Pol J Med Phys Eng 2012;18(1):7-10.

PL ISSN 1425-4689

doi: 10.2478/v10013-012-0002-2

website: http://www.pjmpe.waw.pl

Andrzej Hacura ${ }^{1}$, Wacława Marcoin ${ }^{2}$, Karol Pasterny ${ }^{1}$

\title{
Application of FTIR spectroscopy to study the thermal stability of magnesium aspartate-arginine
}

\author{
${ }^{1}$ Institute of Physics, University of Silesia, Uniwersytecka 4, 40-007 Katowice, Poland \\ ${ }^{2}$ Medical College in Silesia, Mickiewicza 29, 40-085 Katowice, Poland \\ e-mail: andrzej.hacura@us.edu.pl
}

\begin{abstract}
FTIR spectroscopy has been applied to study the thermal stability of magnesium aspartatearginine. An attempt has been made, using theoretically predicted IR spectra, to relate the changes in the experimental spectra with the decomposition process of the studied magnesium complex.
\end{abstract}

Key words: thermal stability, magnesium salts, FTIR spectroscopy

\section{Introduction}

Magnesium belongs to the group of bioelements which show multidirectional biological activity and are necessary for the proper functioning of human organism. Many years of studies on the role of this element in human organism allowed determining exactly the function which it plays in numerous essential processes and metabolic transformations. The method helping the balance of the storage of this element, often called as "metal of life", is to supplement it by drugs containing magnesium. The connections of magnesium with organic ligands are the best for magnesium supplementation. Amino acids are convenient factors modifying functioning of pharmacologically active compounds. Arginine - an amino acid which performs a vital role in blood circulation system (decreases blood viscosity) is often used as such factor.

An important feature of a given drug is its stability both in pharmacological and in economic sense [1]. The so called accelerated ageing test, used frequently in drug stability research [2, 6], is based on kinetic method of measuring the speed of the decomposition reaction in conditions of increased temperature. It corresponds to the process of natural ageing accompanied by changes in the examined samples. The influence of the high temperature allows to observe the occurring changes very fast, in comparison with the same effect obtained in the way of natural ageing. 
IR spectroscopy gives the possibility to observe changes in the samples by analysis of their spectra registered at various conditions and comparison of experimental results with theoretical calculations [3-5]. The aim of our study was to apply this very sensitive IR method, for investigation the thermal stability of magnesium aspartate-arginine.

\section{Experimental}

The absorption spectra of studied magnesium complexes in solid state and in water solutions were obtained using Fourier transform infrared spectrometer Bio-Rad 6000. A single reflection ATR accessory (MIRacle-Pike) with a KRS-5 prism was applied for these measurements. The spectrometer was equipped with DTGS detector and operated with $2 \mathrm{~cm}^{-1}$ resolution in $4000-550 \mathrm{~cm}^{-1}$ wavelength range.

\section{Results and Discussion}

In Figs. 1 and 2, the IR spectra of crystalline magnesium aspartate sample and aqueous solution of magnesium aspartate-arginine are shown, respectively. The application of drugs in the form of aqueous suspension is more convenient from the medical point of view. In the studied temperature range the spectra of solid phase samples of the examined compounds do not exhibit noticeable changes as can be seen in Fig. 1. Spectra of the aqueous solutions are more susceptible to the temperature changes, see Fig. 2.

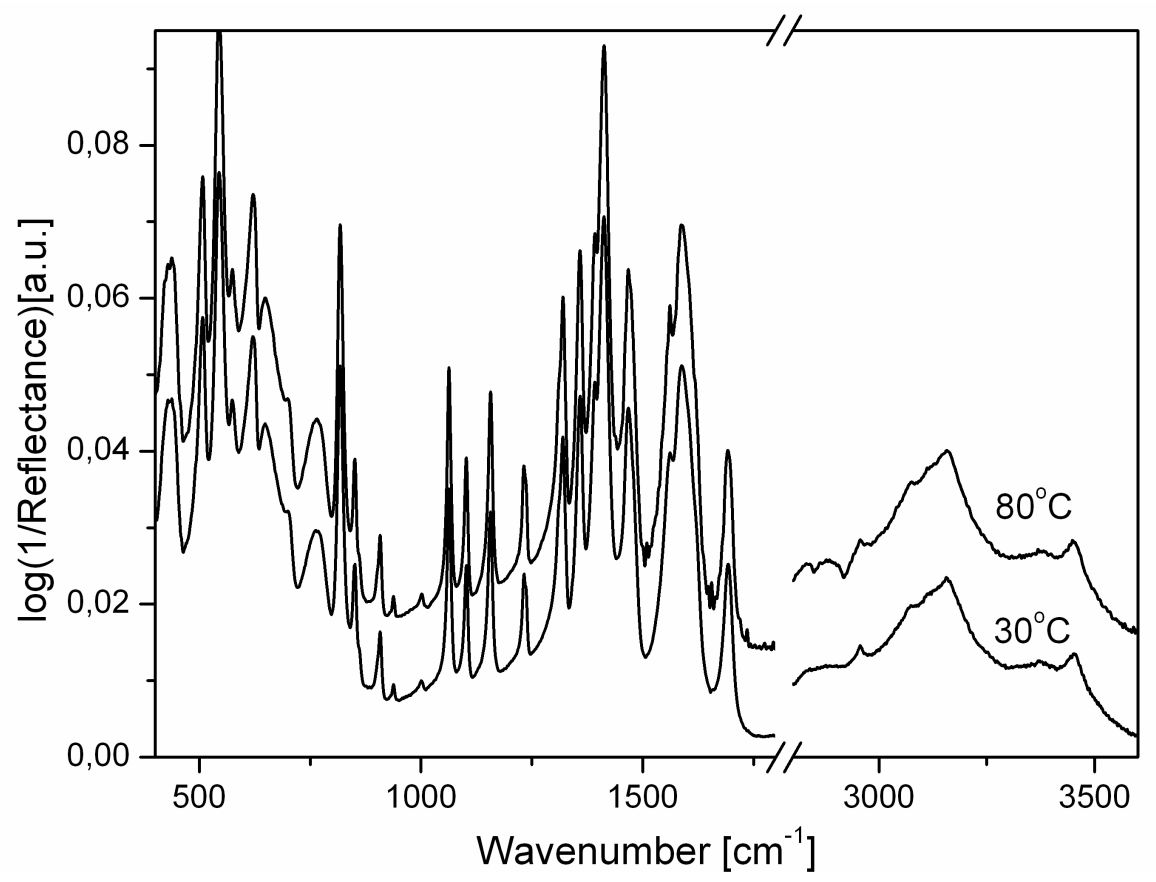

Figure 1. IR spectra of magnesium aspartate in solid state after ageing process at various temperatures.

We focused our analysis on the spectra of the aqueous solution of magnesium aspartate-arginine; in this case the changes in the spectra resulting from the increasing 
temperature were most pronounced. In paper [3] the results of theoretical density functional theory (DFT) calculations of magnesium aspartate - arginine structure and its spectroscopic characteristics along with the approximate description of IR modes have been presented. Different types of changes of the bands with temperature can be observed. For example, the weak $1457 \mathrm{~cm}^{-1}$ band at $70^{\circ} \mathrm{C}$ vanishes almost completely with increasing temperature, i.e. at $90^{\circ} \mathrm{C}$. Similar behaviour is observed in the case of $2852 \mathrm{~cm}^{-1}$ band; its medium intensity at $70^{\circ} \mathrm{C}$ becomes very weak as the temperature increases. Both these bands are associated with the vibrations of hydrogen and carbon atoms located in that part of arginine which is close to the aspartatic acid.

The $1457 \mathrm{~cm}^{-1}$ mode has been ascribed (see paper [3]) to the combination of bending $\mathrm{CH}_{2}$ vibrations and the $2852 \mathrm{~cm}^{-1}$ to the combination of stretching symmetrical $\mathrm{CH}_{2}$ vibrations. The opposite direction of the band intensity changes accompanied by the "aging" process can be observed for the $1092,8 \mathrm{~cm}^{-1}$ band. At $70^{\circ} \mathrm{C}$ there is a lack of a noticeably visible band, at $80^{\circ} \mathrm{C}$ a weak band emerges, passing to a band with medium intensity at $90^{\circ} \mathrm{C}$. This mode has been ascribed [3] to the combination of rocking $\mathrm{NH}_{2}$ and bending $\mathrm{HC}(\mathrm{N})$ vibrations of atoms located at the end of arginine.

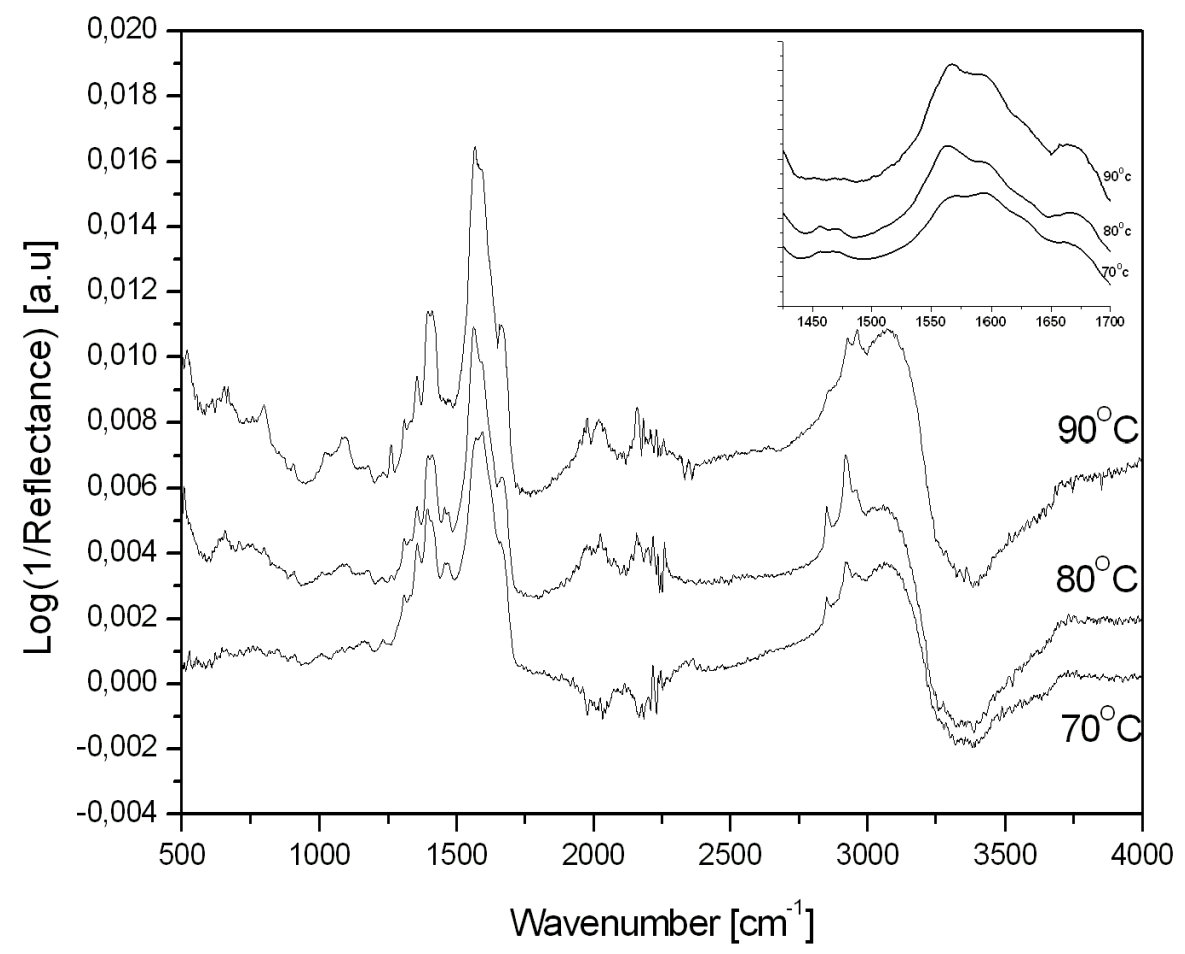

Figure 2. IR spectra of magnesium aspartate-arginine in water solutions after ageing process at various temperatures.

Especially interesting are changes in the two neighboring bands, $1564 \mathrm{~cm}^{-1}$ and $1593 \mathrm{~cm}^{-1}$. At $70^{\circ} \mathrm{C}$ the $1564 \mathrm{~cm}^{-1}$ band is weaker than the $1593 \mathrm{~cm}^{-1}$ one. When the temperature increases, i.e. at 80 and $90^{\circ} \mathrm{C}$, we observe the reversal situation, the band at $1564 \mathrm{~cm}^{-1}$ becomes stronger than that at $1593 \mathrm{~cm}^{-1}$. According to the description given in calculation presented previously [3], both these bands correspond to the bending $\mathrm{NH}_{2}$ vibrations; $1564 \mathrm{~cm}^{-1}$ is associated with the nitrogen atom located in the 
terminal part of the arginine whereas $1593 \mathrm{~cm}^{-1}$ with nitrogen atom in the neighborhood of the aspartatic acid. The observed changes in these bands with increasing temperature can be explained as follows. Due to "aging" (increase in temperature) we can expect, first of all, the decomposition of the magnesium aspartate-arginine into arginine and aspartatic acid [3].

The $\mathrm{NH}_{2}$ group responsible for the $1593 \mathrm{~cm}^{-1}$ vibrations mode in magnesium aspartate-arginine is now in similar surroundigs as $\mathrm{NH}_{2}$ group responsible for the 1564 $\mathrm{cm}^{-1}$ mode causing thereby the growth of its intensity in comparison with intensity of $1593 \mathrm{~cm}^{-1}$ mode. The latter will be still present in the spectrum, because not all molecules undergo decomposition, but with noticeable weaker intensity.

\section{Conclusions}

The analysis of infrared spectra of magnesium aspartate-arginine confirms the better thermal stability of solid samples in comparison with their water solution ones. The IRATR method seems to be very useful for fast qualitative determination of the thermal stability of magnesium salts end their water solutions.

\section{References}

[1]. Carstensen, J.T.,1989. Drug Stability. Principles \& Practices. Marcel Dekker, New York.

[2]. Lin, S-Y., Wang, S-L., 2012. Advances in simultaneous DSC-FTIR microspectroscopy for rapid solid-state chemical stability studies: Some dipeptide drugs as examples. Advanced Drug Delivery Reviews. 64, 461-478.

[3]. Marcoin, W., Pasterny, K.,, Wrzalik, R., 2005. Quantum-mechanical calculations of magnesium aspartate-arginine structure and spectroscopic characteristic. Journal of Molecular Structure. 743, 85-92.

[4]. Marcoin, W., Pasterny, K., Pasterna, G., Wrzalik, R., 2006. Quantum-mechanical calculations and spectroscopic characteristic of magnesium glutamate-glycine and magnesium glutamate-arginine. Journal of Molecular Structure. 792-793, 186-193.

[5]. Marcoin, W., Pasterny, K., Pasterna, G., 2004. Theoretical and experimental studies on magnesium aspartate-glycine. Physica Medica, XX, Suppl. 1, 120-122.

[6]. Wu, L., Zhang, J., Watanabe, W., 2011. Physical and chemical stability of drug nanoparticles. Advanced Drug Delivery Reviews. 63, 456-469. 